\title{
COMPARISON OF TECHNIQUES FOR MEASURING THE ISONIAZID RESISTANCE OF TUBERCLE BACILLI
}

BY

\author{
D. A. MITCHISON* \\ From the Postgraduate Medical School of London
}

(RECEIVED FOR PUBLICATION APRIL 21, 1953)

As with streptomycin and P.A.S., the introduction of isoniazid for the treatment of tuberculosis has raised the problem of the development of resistance to the drug in strains of tubercle bacilli recovered from treated patients. In the Medical Research Council (1952) trials $71 \%$ of cultures from patients who had received three months' treatment with isoniazid alone were found to be resistant. Although combined treatment with isoniazid and streptomycin may markedly reduce this high incidence of resistant strains, it will still be essential to measure the sensitivity of tubercle bacilli recovered from patients before, during, and after treatment.

A number of methods have been used for determining the sensitivity of tubercle bacilli to streptomycin and other chemotherapeutic agents. These can be classified as (1) the direct test, when the sputum concentrate is placed directly on to a series of solid medium slopes containing a range of drug concentrations; (2) the indirect test, when a suspension of a primary culture on drug-free medium is inoculated on to a similar series of solid or fluid media containing a range of drug concentrations; and (3) the subculture test, when the strain is subcultured into liquid medium, usually Dubos Tween-albumin medium, and then the resulting growth is inoculated into tubes of the same medium containing a range of drug concentrations. This last method has been recommended by the Medical Research Council (1948) for streptomycin, since the test can be performed under standardized conditions in a medium with a constant $p \mathbf{H}$ and composition.

During the Medical Research Council trials of isoniazid a series of experiments was carried out to investigate the behaviour of isoniazid-resistant strains in the indirect and subculture tests, since these were thought to be the methods most likely to be adopted in practice.

- In receipt of a grant from the Medical Research Council.

\section{Methods}

Strains.-Isoniazid-resistant strains of tubercle $\vec{\overrightarrow{ }}$ bacilli were obtained from patients in the Medicalo Research Council (1952) trials. Sputum from these patients was cultured on isoniazid-free egg medium $\overrightarrow{\vec{z}}$ slopes. The primary cultures obtained were used in this investigation either immediately after an isoniazid sensitivity test on solid medium had been read or $\vec{c}$ immediately after the culture had become positive. $\omega$ In the latter case the strains were known to be probably resistant because of previous tests on strains from the same patient.

Sensitivity Tests.-The solid medium test has been® described previously (Medical Research Council, $\stackrel{\circ}{\mathbb{Q}}$ 1952 ; Barnett, Bushby, and Mitchison, 1953). Loopfuls of a bacillary suspension from the growth either $\overrightarrow{0}$ on solid or in liquid medium were inoculated on to 3 slopes of Lowenstein medium containing isoniazid in concentrations of $0,0.2,1,5,10$, and $50 \mu \mathrm{g}$. per ml.0 In a few cases slopes containing 200 and $500 \mu \mathrm{g}$. per $\mathrm{ml}$. were also used. The degree of growth was reado after 14 and 28 days' incubation. (The 28-day read-o ing will be referred to except where otherwise indi cated.) Cultures were counted as having grown wheres 20 or more colonies appeared. Dubos Tween-albumin 3 medium was used in liquid medium sensitivity tests as previously described (Barnett et al., 1953). Tubes of this medium, referred to as Dubos medium, con $\frac{D}{8}$ tained a range of isoniazid concentrations increasing: by twofold steps from 0.012 to $100 \mu \mathrm{g}$. per ml. togethen with a drug-free control tube. The same medium? without Tween was also used. Tests were read aftef $7,10,14,21$, and 28 days' incubation.

The isoniazid-sensitive strain $\mathrm{H} 37 \mathrm{Rv}$ was always set up under identical conditions with any batch of resistant strains.

\section{Results}

Experiment 1.-Sensitivity tests on Lowenstein medium were set up from the primary cultures of 18 resistant strains and three sensitive strain obtained before treatment with isoniazid. These were then subcultured once in Lowenstein mediurf and twice in Dubos medium. Sensitivity tests is 
Dubos medium and on Lowenstein medium were set up together from the final culture in Dubos medium. The results have, in part, been described previously (Barnett et al., 1953). After subculture the degree of resistance of highly resistant strains was shown usually to have fallen markedly, as measured in Lowenstein medium tests. Thus, out of 12 strains inhibited by 50 or $>50 \mu \mathrm{g}$. per ml. isoniazid before subculture, 10 were inhibited by 0.2 to $10 \mu \mathrm{g}$. per ml. after subculture. On the other hand, four strains originally inhibited by 5 or 10 $\mu \mathrm{g}$. per $\mathrm{ml}$. maintained their degree of resistance during subculture.

As shown in Fig. 1 there was a good correlation between the results of the tests on Lowenstein

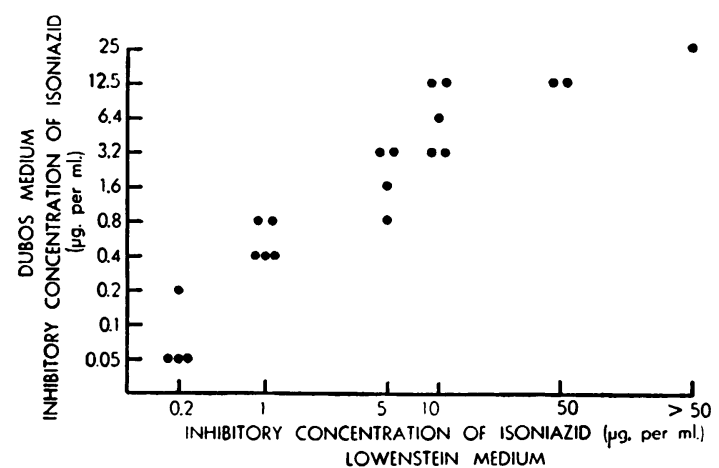

FIG. 1.-Correlation between the minimal inhibitory concentrations of isoniazid in Lowenstein and in Dubos medium with 18 resistant and three sensitive strains grown in Dubos medium. Period of incubation, 28 days on Lowenstein slopes and 14 days in Dubos medium.

medium and those in Dubos medium read after 14 days' incubation. The 14-day readings of the tests in Dubos medium were compared with those on solid medium, for two reasons. First, they yielded a better correlation than did seven-, 10-, 21-, or 28-day readings. Secondly, they would be expected to yield similar results from consideration of the nu nbers of bacilli involved. Cultures in the same Dubos medium have been found to contain about $2 \times 10^{7}$ viable units per ml. (Barnett et al., 1953). They were inoculated on to the slopes with a loop containing $0.005 \mathrm{ml}$. In the Lowenstein tests growth was counted as occurring where 20 or more colonies appeared. Therefore for resistant organisms to produce more than 20 colonies on a slope containing isoniazid they should be present in a proportion to the remainder of the strain of at least 1:5,000. Now, using the same Dubos medium and inoculum size and artificial mixtures of streptomycin-sensitive and resistant strains, Mitchison (1950) has shown that the proportion of resistant to sensitive bacilli necessary to cause a streptomycin sensitivity test in Dubos medium to be read as resistant after 14 days' incubation varies from $1: 440$ to $1: 180,000$. By analogy, therefore, one would expect that the 14-day readings would correspond best with the results of the Lowenstein tests.

The average inhibitory concentration of isoniazid in Lowenstein medium seemed to be slightly higher than in Dubos medium, particularly with the more highly resistant strains. Thus strains inhibited on Lowenstein medium by $1,5,10,50$, and more than $50 \mu \mathrm{g}$. per $\mathrm{ml}$. were inhibited in Dubos medium on the average by respectively 0.53 , $1.9,6.3,12.5$, and $25 \mu \mathrm{g}$. per $\mathrm{ml}$. This is unlikely to be due to the choice of the 14-day reading in the Dubos medium tests since, where tests were set up on Lowenstein slopes containing higher concentrations of isoniazid, the most highly resistant strains were inhibited by 200 , or more rarely 500 , $\mu$ g. per ml., whereas in Dubos medium tests such strains were usually inhibited by only 25 or $50 \mu$ g. per $\mathrm{ml}$. even when incubation was prolonged to 28 days.

Experiment 2.-Suspensions of bacilli in distilled water were prepared from a series of 24 primary resistant cultures which had been stored for about a month at room temperature after first appearing positive. These suspensions, which were of about the same opacity as a fully grown Dubos medium culture, were inoculated directly on to Lowenstein medium sensitivity ${ }^{\circ}$ tests and into Dubos medium tests both with and without Tween.

The differences between the minimal inhibitory concentrations in Dubos medium with and without

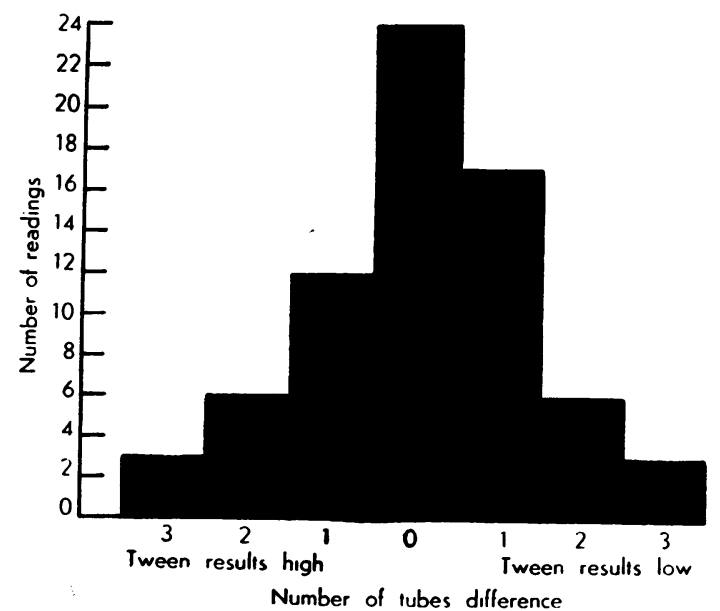

FIG. 2.-The effect of Tween in Dubos medium tests on resistant strains. Readings of tests were taken after 14, 21, and 28 days. The differences between the inhibitory end-points found in medium with and without Tween is indicated. 
Tween read after 14,21 , and 28 days' incubation are shown in Fig. 2. The presence of Tween did not appear to influence the readings, since there was an equal balance between readings which were higher and those which were lower in medium with Tween.

The results of the sensitivity tests on Lowenstein medium and in Dubos medium are shown in Fig. 3 for 22 of the resistant strains and five sensitive strains. It is apparent that there was a very poor correlation between the end-points found by the two methods. Thus, of nine strains yielding confluent growth or innumerable colonies on slopes containing $50 \mu \mathrm{g}$. per $\mathrm{ml}$., six were inhibited by $6.4 \mu \mathrm{g}$. per ml. or less in Dubos medium after 14 days' incubation. These low readings were not due

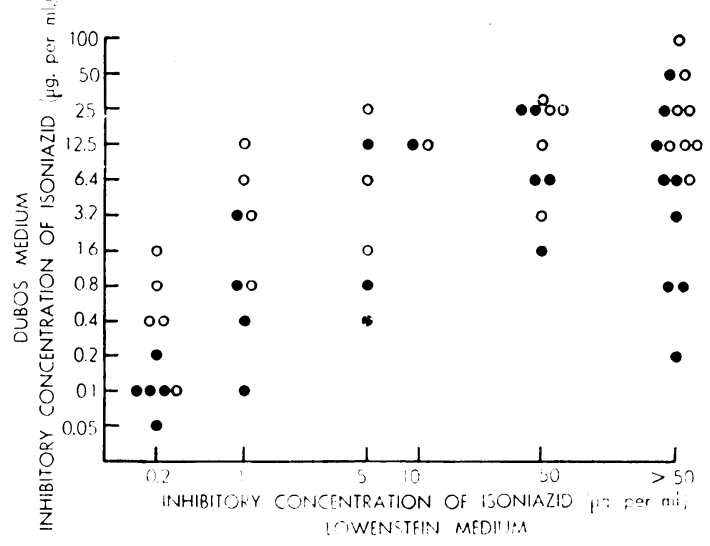

FIG. 3.-Correation between the minimal inhibitory concentrations of isoniazid in Lowenstein and in Dubos medium with 22 resistant and five sensitive strains grown on Lowenstein slopes. Closed circles $=$ Dubos medium tests read after 14 days; open circles - Dubos medium tests read after 28 days. In both cases the Lowenstein medium tests were read after 28 days.

to slow growth of the whole population of the strain, since in each case there was growth in the control tube of the sensitivity test after seven days' incubation. After 28 days' incubation growth appeared in tubes containing higher concentrations of isoniazid and also in a number of strains having a lower degree of resistance on solid medium.

The tenth strain (not shown in Fig. 3) that was highly resistant on solid medium did not grow in Dubos medium with or without Tween, except in the tube containing $0.05 \mu \mathrm{g}$. per $\mathrm{ml}$. in the series with Tween, and in the tube containing $0.012 \mu \mathrm{g}$. per $\mathrm{ml}$. in the series without Tween. One of the strains inhibited by $1 \mu \mathrm{g}$. per $\mathrm{ml}$. on Lowenstein medium yielded no growth in Dubos medium after 10 days' incubation, and the growth after this was irregular. Behaviour in Dubos medium without Tween 80 was similar. With six more

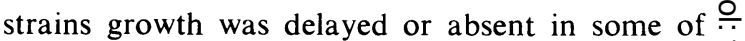
the tubes in the Dubos sensitivity tests lying within $\overrightarrow{\vec{D}}$ a range of others in which growth had occurred.

On the other hand, one strain (not shown in Fig. 당 3 ), which grew well in Dubos medium, did not $\frac{5}{\omega}$ grow on the Lowenstein slopes, and in two of $\frac{\vec{\phi}}{\alpha}$ the Lowenstein medium tests on highly resistant $\frac{2}{0}$ strains a single slope $(0.2$ and $1 \mu \mathrm{g}$. per ml. $)$ yielded no growth.

These results suggest that resistant strains growing on Lowenstein medium did not grow satis- $\vec{\sigma}$ factorily when subcultured into Dubos medium, particularly when they were highly resistant. The? eventual growth in higher isoniazid concen-i trations in Dubos medium after 28 days' incu- bation would appear to be due either to only a very $\vec{\infty}$

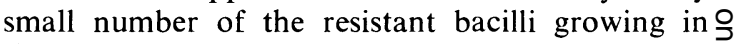
the inoculum or to a much slower rate of growth of highly resistant organisms than of those which 3 had a lower degree of resistance or were sensitive. A much smaller number of strains grew in Dubos $\vec{\varphi}$ medium, but failed to grow where expected on all $\mathrm{W}$ or one of the Lowenstein slopes.

Experiment 3.-Primary cultures of sputum from patients known to have produced resistant strains previously were examined at weekly intervals. Within three days of the first appearance $\frac{}{\Phi}$ of growth a Lowenstein medium sensitivity test $\stackrel{2}{\vec{F}}$ was set up in the usual manner and also tests with윽 the inoculum diluted $1: 10$ and $1: 100$. The original primary culture was then incubated at $37^{\circ} \mathrm{C}$. and further sensitivity tests carried out after 14 and 28 days. Finally a sensitivity test was set up fromo the control slope of the first sensitivity test, i.e.o a subculture, which had been incubated for 28 . days.

The results are given in Table I. As might be expected, the inocu'um size used in the test? influenced the apparent degree of resistance of the strains, since the mean of the end-points was 19.9 ? $\mu \mathrm{g}$. per $\mathrm{ml}$. with the usual inoculum, $9.9 \mu \mathrm{g}$. per $\mathrm{ml}$. when this was diluted $1: 10$, and $5.6 \mu \mathrm{g}$. per $\mathrm{ml}$. when diluted $1: 100$. In calculating the means $\tilde{O}$ strains growing on $50 \mu \mathrm{g}$. per $\mathrm{ml}$. were countect as being inhibited by $300 \mu \mathrm{g}$. per ml., sincer this has been found in other experiments t\& be the average inhibitory end-point where suck strains have been titrated at higher isoniazid cono? centrations. The fall in the inhibitory end-pointo with decreasing inoculum size was marked in some्ब. strains and absent in others, due presumably t\$ differences in their degree of homogeneity.

Incubating the primary cultures caused aß irregular decrease in the apparent degree of resistance. The fall in resistance was not due to 
TABLE I

EFFECT OF STORAGE AT $37^{\circ} \mathrm{C}$., INOCULUM SIZE, AND SUBCULTURE ON INHIBITORY CONCENTRATION OF ISONIAZID IN LOWENSTEIN MEDIUM TESTS ON RESISTANT STRAINS $(\mu \mathrm{g} . / \mathrm{ml}$. $)$

\begin{tabular}{|c|c|c|c|c|c|c|}
\hline \multirow{4}{*}{ Strain } & \multicolumn{5}{|c|}{$\begin{array}{l}\text { Period of Storage at } 37^{\circ} \mathrm{C} \text {. after Culture } \\
\text { Became Positive (Days) }\end{array}$} & \multirow{4}{*}{$\begin{array}{l}\text { Sub- } \\
\text { culture }\end{array}$} \\
\hline & & 0 & & 14 & 28 & \\
\hline & \multicolumn{3}{|c|}{ Dilution of Inoculum } & & & \\
\hline & $\begin{array}{l}\text { Undi- } \\
\text { luted }\end{array}$ & $1: 10$ & $1: 100$ & & & \\
\hline $\begin{array}{r}1 \\
2 . \\
3 \\
4 \\
5 \\
6 \\
7 \\
8 \\
9 \\
10 \\
11 \\
12 \\
13\end{array}$ & $\begin{array}{r}>50 \\
>50 \\
>50 \\
>50 \\
>50 \\
10 \\
5 \\
5 \\
5 \\
5 \\
5 \\
1 \\
1\end{array}$ & $\begin{array}{r}>50 \\
>50 \\
>50 \\
50 \\
10 \\
5 \\
5 \\
5 \\
5 \\
1 \\
1 \\
1 \\
1\end{array}$ & $\begin{aligned}>50 \\
>50 \\
10 \\
>50 \\
5 \\
5 \\
5 \\
5 \\
5 \\
1 \\
(0 \cdot 2) * \\
1 \\
0 \cdot 2\end{aligned}$ & $\begin{array}{c}>50 \\
>50 \\
50 \\
50 \\
5 \\
5 \\
5 \\
5 \\
5 \\
1 \\
(0 \cdot 2) * \\
1 \\
1\end{array}$ & $\begin{array}{r}>50 \\
>50 \\
>50 \\
>50 \\
5 \\
50 \\
5 \\
5 \\
5 \\
1 \\
0 \cdot 2 \\
1 \\
1\end{array}$ & $\begin{array}{c}>50 \\
10 \\
50 \\
5 \\
5 \\
5 \\
5 \\
5 \\
5 \\
1 \\
5 \\
0 \cdot 2 \\
0.2\end{array}$ \\
\hline $\begin{array}{l}\text { Geometric } \\
\text { mean }\end{array}$ & $19 \cdot 9$ & $9 \cdot 9$ & $5 \cdot 6$ & $8 \cdot 3$ & $14 \cdot 0$ & $4 \cdot 6$ \\
\hline $\begin{array}{l}\text { Poor growth } \\
\text { on control } \\
\text { slopes } † \text { after } \\
\text { incubation } \\
\text { (days) }\end{array}$ & & & & & & \\
\hline $\begin{array}{l}14 \\
28\end{array}$ & $\begin{array}{l}2 \\
2\end{array}$ & $\begin{array}{l}5 \\
2\end{array}$ & $\begin{array}{r}13 \\
2\end{array}$ & $\begin{array}{l}1 \\
1\end{array}$ & $\begin{array}{l}0 \\
0\end{array}$ & 1 \\
\hline
\end{tabular}

* See Table II.

t The last two lines of the table indicate the number of tests in which the degree of growth on the control tube was less than the usual confluent growth. This gives an indication of the viability of the strain. The results are given when the tests had been incubated for 14 and 28 days.

small viable inoculum in the tests, since the control tubes showed, if anything, a greater degree of growth than the control tubes in the first test (see the bottom two lines in Table I). In one strain (Table I, strain 11) whose results are given in detail in Table II there is evidence that the strain was initially composed of a mixture of resistant and sensitive organisms. After 14 days' incubation

TABLE II

RESULTS OF LOWENSTEIN MEDIUM SENSITIVITY TESTS ON STRAIN 11

\begin{tabular}{|c|c|c|c|c|c|c|}
\hline & \multicolumn{6}{|c|}{ Isoniazid Concentration $(\mu \mathrm{g}$. per $\mathrm{ml}$.) } \\
\hline & 0 & 0.2 & 1 & 5 & 10 & 50 \\
\hline 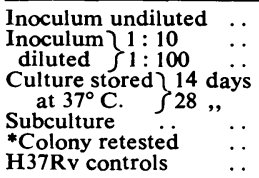 & $\begin{array}{l}\text { IC } \\
20-100 \\
16 \\
1 * \\
\text { CG } \\
\text { CG } \\
\text { CG } \\
\text { CG }\end{array}$ & $\begin{array}{c}10 \\
20-100 \\
2 \\
0 \\
0 \\
\text { IC } \\
0 \\
0\end{array}$ & $\begin{array}{c}20-100 \\
1 \\
0 \\
0 \\
0 \\
20-100 \\
0 \\
0\end{array}$ & $\begin{array}{l}3 \\
0 \\
0 \\
0 \\
0 \\
8 \\
0 \\
0\end{array}$ & $\begin{array}{r}6 \\
0 \\
0 \\
0 \\
0 \\
13 \\
0 \\
0\end{array}$ & $\begin{array}{r}1 \\
0 \\
0 \\
0 \\
0 \\
0 \\
10 \\
0 \\
0\end{array}$ \\
\hline
\end{tabular}

$\mathrm{CG}=$ confluent growth. IC $=$ innumerable colonies. Numbers indicate numbers of colonies. the resistant organisms had apparently died and were replaced at 28 days by a growth of sensitive organisms. The test on the subculture, on the other hand, showed that this population still contained resistant organisms. In another strain (Table I, strain 5), the original tests indicated that the majority of the population was inhibited by $5 \mu \mathrm{g}$. per ml., while there was a considerable fraction of highly resistant organisms yielding, with the undiluted inoculum, innumerable colonies or 20 to 100 colonies on slopes containing 5,10 , and 50 $\mu \mathrm{g}$. per $\mathrm{ml}$. This highly resistant fraction did not appear in subsequent tests, although the amount of growth on the control tubes indicated that there had been no marked fall in the number of viable organisms in the inocula. Here again it would appear that a proportion of the strain had lost its viability during incubation.

There also seemed to be an irregular but definite decrease in resistance after subculture, the average degree of resistance being $19.9 \mu \mathrm{g}$. per ml. before subculture and $4.6 \mu \mathrm{g}$. per $\mathrm{ml}$. after subculture. This occurred in strains with all degrees of resistance and again did not appear to be due to a decrease in the inoculum. One must conclude that some of the resistant portions of the populations did not survive the subculture.

\section{Discussion}

In Experiment 1 the Lowenstein medium tests carried out before and after subculture showed that there had been a large and irregular fall in the degree of resistance of the strains tested. The alteration in the bacterial population that must have occurred took place during one subculture on Lowenstein medium and two in Dubos medium.

The results of simultaneous tests in Lowenstein and in Dubos medium carried out in Experiments 1 and 2 provide some information as to the nature of this change. In both experiments the average concentrations that inhibited growth were lower in Dubos than in Lowenstein medium. This may, perhaps, have been due to binding or destruction of the isoniazid in the Lowenstein medium but was not due to the presence of Tween in the Dubos medium (Fig. 2). However, in Experiment 2 (Fig. 3), where the inoculum had been grown on Lowenstein slopes, the strains inhibited by any one concentration in Lowenstein medium were inhibited by a very wide range of concentrations in Dubos medium. This seemed to be due mainly to some of the more resistant components of strains which were able to grow on 10 $\mu \mathrm{g}$. per $\mathrm{ml}$. or more isoniazid in Lowenstein 
slopes failing to grow in corresponding concentrations in Dubos medium. It appears unlikely that this was due to the use of solid medium in one test and liquid medium in the other, since there was a reasonably good correlation between the results carried out simultaneously by these two methods in Experiment 1. In this experiment the inoculum had been grown in Dubos medium. It must, however, be admitted that only three strains containing these highly resistant components were tested.

Thus it seems that resistant strains sometimes failed to grow in Dubos medium when the inoculum had been grown on Lowenstein medium, whereas they grew more uniformly when the inoculum had been taken from subcultures in Dubos medium. This suggests that the strains had become adapted to Dubos medium during subculture in it. That such an adaptation occurs was also indicated by previous work (Barnett et al. 1953) in which variants of H37Rv, while being made resistant by passages in Dubos medium containing isoniazid, were found to have poor viability during their first few passages, but not subsequently.

Fisher (1952) has shown that a highly resistant variant of H37Rv grew well in Dubos medium and had a growth factor requirement present in the bovine fraction $\mathrm{V}$ albumin of this medium. Fraction $\mathrm{V}$ albumin, a partially purified product, may contain only small amounts of this factor, and Fisher's strain, having been produced in Dubos medium, may have already become adapted to suboptimal concentrations of it. It is possible that the more complex Lowenstein medium may contain larger amounts of the factor and thus be a more suitable medium for the initial growth of resistant strains.

Although Dubos medium did not seem as satisfactory as Lowenstein medium for the growth of recently isolated resistant strains, Lowenstein medium was itself not entirely satisfactory, since one strain in Experiment 2 grew in Dubos medium but did not grow on Lowenstein medium, and in both Experiments 2 and 3 several Lowenstein slopes did not yield the growth that might be expected. It should be noted that this did not cccur in the Lowenstein tests on strains growing in Dubos medium in Experiment 1. This irregular viability was not due to a single unsatisfactory batch of medium, since several batches were used in each experiment, and it has been observed in other unreported experiments. A better medium might be evolved by the addition of an excess of Fisher's factor.
The fall in resistance that occurred during subculture in Experiment 1 was only shown by strains that were highly resistant. It appears probable that it took place mainly during the establishment of the strains in Dubos medium but partly also during their subculture on Lowenstein medium as shown in Experiment 3. The final level of resistance attained by these strains would then be determined by organisms with a lower degree of resis- $\vec{c}$ tance and greater viability in the original population outgrowing the highly resistant organisms during the three subcultures.

It seems clear that subculturing, particularly in Dubos medium, should be avoided in any method i for the routine testing of isoniazid resistance. In $\overrightarrow{\vec{A}}$ particular the subculture test recommended for $\vec{\infty}$ streptomycin sensitivity tests by the Medical 음 Research Council (1948) and the method of Collins $\rightarrow$ and Bowen (1953) should not be used. The direct test, in which Lowenstein slopes containing a range of isoniazid concentrations are inoculated $\vec{\oplus}$ with a sputum concentrate, involves no subcultures but is only possible when there are many tubercle $\square$ bacilli in the sputum. Furthermore, the incubation period before growth appears is variable in primary cultures, and during this period much of the isoniazid may have been destroyed (Knox, King, and Woodroffe, 1952 ; Mitchison, 1952).

Although no method is entirely satisfactory, the indirect test recommended by the Medical Research Council (1952) seems the best compromise, since it involves one culture on Lowenstein medium before testing. The results of Experiment 3 suggest that the inoculum should be approximately standardized and that the test should be carried out as soon as possible after the primary culture has yielded visible growth.

\section{Summary}

Isoniazid-resistant strains of tubercle bacilli were $\bar{N}$ tested simultaneously on Lowenstein medium and $\sigma$ in Dubos medium. When the inoculum was 0 obtained from growth on Lowenstein medium $\underset{\omega}{N}$ there was a poor correlation between the results, 0 suggesting that highly resistant strains grew 0 poorly in Dubos medium. When the inoculum was obtained from cultures in Dubos medium there $\stackrel{\mathcal{Q}}{+}$ was good agreement between the results. It is 7 postulated that resistant strains may have a growth factor requirement and that during subculture in $\stackrel{\odot}{\circ}$ Dubos medium they may become adapted to $\stackrel{\mathbb{Q}}{\mathbb{Q}}$ growth in suboptimal concentrations of this factor. Lowenstein medium, although more satisfactory than Dubos medium for the growth of resistanto 
strains, was itself not entirely satisfactory, since in some tests growth did not occur where it was to be expected.

Although the best method of performing isoniazid sensitivity tests has not been found, it is suggested that strains obtained on isoniazid-free egg medium should be tested by inoculating them on to Lowenstein slopes containing a range of isoniazid concentrations. Using such a procedure, it was found that storage of the primary culture at $37^{\circ} \mathrm{C}$., dilution of the inoculum, and subculture all resulted in a fall in the degree of resistance. In some cases this was thought to be due to the selective mortality of the resistant component in a heterogeneous bacterial population.

Miss M. Monk has given valuable technical assistance throughout this investigation.

The isoniazid was kindly supplied as "nydrazid" by Messrs. E. R. Squibb \& Sons, London.

\section{REFERENCES}

Barnett, M., Bushby, S. R. M., and Mitchison, D. A. (1953). Lancet. 1,314 .

Collins, D. M., and Bowen, D. A. L. (1953). Tubercle, 34, 8.

Collins, D. M., and Bowen, D. A. L. (1953). Tuberch

Knox, R., King, M. B., and Woodroffe, R. C. (1952). Lancet, 2, 854. Medical Research Council (1948). Ibid., 2, 862.

(1952). Brit. med. J., 2, 735 .

Mitchison, D. A. (1950). Thorax, 5, 162.

(1952). Lancet, 2, 858. 